\title{
A 70-year-old woman with heart failure with preserved ejection fraction
}

\author{
Prashant Sharma MD, Vijaiganesh Nagarajan MD \\ See also page 518 and www.cmaj.ca/lookup/doi/10.1503/cmaj.140430
}

Competing interests: None declared.

This article has been peer reviewed.

Correspondence to: Prashant Sharma, sharma. prashant@mayo.edu

CMAJ 2015. DOI:10.1503 /cmaj.131742
A 70-year-old woman was referred to her family physician by the emergency department for follow-up of shortness of breath, orthopnea and swelling of her legs that she had experienced for two months. She had no other symptoms and was taking amlodipine $10 \mathrm{mg}$ daily and lisinopril $10 \mathrm{mg}$ daily for hypertension. On physical examination, her blood pressure was $160 / 92 \mathrm{~mm} \mathrm{Hg}$ and pulse rate was 70 beats/min. Estimated central venous pressure was 12 (normal $\leq 8$ ) $\mathrm{cm} \mathrm{H}_{2} \mathrm{O}$. Cardiac examination was unremarkable, and there were bibasilar crackles on lung auscultation. She had bilateral pedal pitting edema. In the emergency department, test results for electrolyte levels and renal function were within normal limits. An electrocardiogram showed sinus rhythm and left ventricular hypertrophy. An echocardiogram showed an ejection fraction of $56 \%$, concentric left ventricular hypertrophy with no substantial valvular abnormalities, and grade III diastolic dysfunction.

\section{What is the diagnosis?}

Based on the presenting symptoms and examination findings, the clinical syndrome of heart failure was diagnosed. Heart failure is a clinical diagnosis. Once the diagnosis is made, ejection fraction measured by echocardiography helps to distinguish between different types of heart failure (Box 1). ${ }^{1}$

Diagnosis of heart failure with preserved

\begin{tabular}{|ll|}
\hline Box 1: Types of heart failure based on left ventricular ejection fraction \\
\hline Ejection fraction, \% & Diagnosis in patients with clinical heart failure \\
\hline$\geq 50$ & \begin{tabular}{l} 
Heart failure with preserved ejection fraction \\
Heart failure with borderline preserved ejection \\
fraction \\
\hline $41-49$
\end{tabular} \\
\hline$\leq 40$ & Heart failure with reduced ejection fraction \\
\hline
\end{tabular}

ejection fraction is challenging, because other potential causes of symptoms have to be excluded. Most patients with heart failure with preserved ejection fraction have evidence of abnormal left ventricular diastolic function on Doppler echocardiography. ${ }^{1,2}$ In the past, heart failure with preserved ejection fraction was commonly called "diastolic heart failure." Because left ventricular diastolic dysfunction is seen not only in patients with heart failure with preserved ejection fraction, but also in those with heart failure with reduced ejection fraction, "heart failure with preserved ejection fraction" has replaced "diastolic heart failure." patients with clinical heart failure have heart failure with preserved ejection fraction. ${ }^{1}$ Among the patients admitted to hospital with decompensated heart failure, the proportion of those with heart failure with preserved ejection fraction has been increasing over the last 15 years. ${ }^{4,5}$

Heart failure with preserved ejection fraction and heart failure with reduced ejection fraction are two distinct syndromes and do not represent a continuous spectrum of disorder. They differ in several aspects, including pathophysiology, patient population and treatment modalities. ${ }^{1,2}$ Pathophysiology of heart failure with preserved ejection fraction is related to diastolic dysfunction, and major predictors are left ventricular relaxation and stiffness. ${ }^{2,3}$

Our patient had clinical features of heart failure but had normal left ventricular ejection fraction, and therefore was given the diagnosis of heart failure with preserved ejection fraction.

\section{What risk factors may have contributed to heart failure in this patient?}

The major predictors of heart failure with preserved ejection fraction are hypertension, atrial fibrillation, older age, female sex, coronary artery disease, obesity, diabetes and hyperlipidemia. ${ }^{1}$ Of these risk factors, hypertension is the most 
frequent, with prevalence of up to $90 \% .^{1,2}$ Also, heart failure with preserved ejection fraction is associated with multiple noncardiac comorbidities, such as chronic kidney disease, lung disease, anemia, liver disease and thyroid diseases.

Our patient had a history of long-standing hypertension, which likely contributed to the development of heart failure with preserved ejection fraction.

\section{What treatment options should be considered for this patient?}

Treatment for patients with heart failure with preserved ejection fraction mainly involves riskfactor modification and treatment of associated comorbidities. ${ }^{1,2}$ Because trial data are limited, strict control of blood pressure and other comorbidities remain the mainstay of management. ${ }^{1,2}$ To date, no treatment has been shown to improve mortality in these patients.

Although angiotensin-converting enzyme inhibitors and $\beta$-blockers have not shown a mortality benefit in patients with heart failure with preserved ejection fraction, they should be used if indicated for comorbid conditions, such as hypertension, coronary artery disease and chronic kidney disease. ${ }^{1}$ Because hypertension, tachycardia and coronary ischemia could cause decompensation of heart failure, these conditions should be treated according to guidelines, including coronary intervention if needed. ${ }^{1-3}$

Diuretics are used for symptomatic patients with volume overload. In the recently reported Treatment of Preserved Cardiac Function Heart Failure with an Aldosterone Antagonist (TOPCAT) study, spironolactone did not decrease mortality, but was noted to decrease the rate of hospital admissions for heart failure. ${ }^{6}$

Lifestyle modification with exercise training has been shown to improve quality of life in these patients. ${ }^{2,7}$ Adherence to medication should be emphasized, and patients with multiple comorbidities should be enrolled in programs for heart failure management if available. ${ }^{1,2}$

What follow-up does this patient require? Patients with heart failure with preserved ejection fraction should receive follow-up like those with heart failure with reduced ejection fraction. Although mortality for heart failure with pre- served ejection fraction may be lower than that for heart failure with reduced ejection fraction, studies show that the rate of heart failure-related hospital admissions among patients with heart failure with preserved ejection fraction is similar to the rate among patients with heart failure with reduced ejection fraction. ${ }^{2,8}$ An efficient system of coordinated care, active patient education and appropriate follow-up is recommended for all patients with heart failure with preserved ejection fraction. ${ }^{1,2}$

\section{Case revisited}

The patient was advised to increase her lisinopril dose to achieve better blood pressure control, and she was also started on spironolactone. At two weeks' follow-up, her shortness of breath was better and her blood pressure was 138/82 $\mathrm{mm} \mathrm{Hg}$. She was referred to a heart failure clinic for follow-up.

\section{References}

1. Yancy CW, Jessup M, Bozkurt B, et al. 2013 ACCF/AHA guideline for the management of heart failure: a report of the American College of Cardiology Foundation/American Heart Association Task Force on Practice Guidelines. J Am Coll Cardiol 2013; 62:e147-239.

2. McMurray JJ, Adamopoulos S, Anker SD, et al. ESC guidelines for the diagnosis and treatment of acute and chronic heart failure 2012: The Task Force for the Diagnosis and Treatment of Acute and Chronic Heart Failure 2012 of the European Society of Cardiology. Developed in collaboration with the Heart Failure Association (HFA) of the ESC. Eur J Heart Fail 2012;14:803-69.

3. Borlaug BA, Paulus WJ. Heart failure with preserved ejection fraction: pathophysiology, diagnosis, and treatment. Eur Heart J 2011;32:670-9.

4. Owan TE, Hodge DO, Herges RM, et al. Trends in prevalence and outcome of heart failure with preserved ejection fraction. N Engl J Med 2006;355:251-9.

5. Steinberg BA, Zhao X, Heidenreich PA, et al. Trends in patient hospitalized with heart failure and preserved left ventricular ejection fraction: prevalence, therapies, and outcomes. Circulation 2012;126:65-75.

6. Pitt B, Pfeffer MA, Assmann SF, et al. Spironolactone for heart failure with preserved ejection fraction. N Engl J Med 2014;370: 1383-92.

7. Kitzman DW, Brubaker PH, Morgan TM, et al. Exercise training in older patients with heart failure and preserved ejection fraction: a randomized, controlled, single-blind trial. Circ Heart Fail 2010;3:659-67.

8. Quiroz R, Doros G, Shaw P, et al. Comparison of characteristics and outcomes of patients with heart failure preserved ejection fraction versus reduced left ventricular ejection fraction in an urban cohort. Am J Cardiol 2014;113:691-6.

Affiliations: Department of Hospital Internal Medicine (Sharma), Mayo Clinic, Rochester, Minn.; Department of Cardiovascular Medicine (Nagarajan), University of Virginia, Charlottesville, Va.

Contributors: Both of the authors equally contributed to the drafting and revision of the manuscript, and gave final approval of the version submitted for publication. 